\title{
Tiliroside is a new potential therapeutic drug for osteoporosis in mice
}

Kai Li ${ }^{1,3 \#}$, Yu Xiao ${ }^{1,3 \#}$, Ziyi Wang ${ }^{2}$, Fangsheng $\mathrm{Fu}^{1}$, Siyuan Shao ${ }^{1}$, Fangming Song ${ }^{1,2}$, Jinmin Zhao ${ }^{1,3}$, Xixi $\operatorname{Lin}^{1}$, Qian Liu, ${ }^{1,}$, Jiake $\mathrm{Xu}^{1,2^{*}}$

1 Research Centre for Regenerative Medicine, Guangxi Key Laboratory of Regenerative Medicine, Guangxi Medical University, Nanning, Guangxi, China 2 School of Biomedical Sciences, the University of Western Australia, Perth, Western Australia, Australia

3 Department of Trauma Orthopedic and Hand Surgery, The First Affiliated Hospital of Guangxi Medical University, Nanning, Guangxi, China

\#Co-first author: Kai Li and Yu Xiao contributed equally to this work

*Correspondence:

Jiake Xu, School of Biomedical Sciences, University of Western Australia, Perth 6009, WA, Australia.

Email: jiake.xu@uwa.edu.au

\section{Funding information:}

The National Natural Science Foundation of China (81501910), the Natural Science Foundation of Guangxi Province (2015GXNSFCA414001，2015GXNSFDA139019), the Guangxi Collaborative Innovation Center for Biomedicine talent cultivation (GCICB-TC-2017001). This study was also supported in part by grants from the Australian Health and Medical Research Council (NHMRC, APP1107828, APP1027932, APP1163933). 


\section{ABSTRACT}

Osteoporosis is a class of metabolic bone disease caused by complexed ramifications. Over-activation of osteoclasts due to a sudden decreased estrogen level plays a pivotal role for postmenopausal women suffering from osteoporosis. Therefore, inhibiting osteoclast formation and function has become a major direction for the treatment of osteoporosis. Tiliroside (Tle) is a salutary dietary glycosidic flavonoid extracted from Oriental Paperbush flower, which has been reported to have an anti-inflammation effect. However, whether Tle affects the osteoclastogenesis and bone resorption remains unknown. Herein, we demonstrate that Tle prevents bone loss in ovariectomy $(\mathrm{OVX})$ in mice and inhibits osteoclast differentiation and bone resorption stimulated by receptor activator of nuclear factor kappa-B ligand (RANKL) in vitro. Molecular mechanism studies reveal that Tle reduces RANKL-induced activation of mitogen-activated protein kinase (MAPK) and NFATc1 pathways, and osteoclastogenesis-related marker genes expression, including Cathepsin K (Ctsk), Matrix metalloproteinase 9 (Mmp9), TRAcP (Acp5), Atp6v0d2. Our research indicates that Tle suppresses osteoclastogenesis and bone loss by down-regulating the RANKL mediated signalling protein activation and expression. In addition, Tle inhibited intracellular reactive oxygen species (ROS) generation which was related to the formation of osteoclasts. Therefore, Tle might serve as a potential drug for osteolytic disease such as osteoporosis.

KEY WORDS: Osteoporosis; Tiliroside; MAPK; Osteoclast differentiation 


\section{1 | INTRODUTION}

Osteoporosis has become an important threat to human health, especially in the aging populations (Reventlow and Bang, 2006; Yoshimura et al., 2010). Osteoporosis is a class of metabolic bone disease mainly caused by enhanced bone resorption and weakened bone formation (Bono and Einhorn, 2003). Decreased estrogen in postmenopausal women can unbalance the coordination between bone resorption function of osteoclasts and osteogenic function of osteoblasts, because the reduction of estrogen level leads to the excessive activation of osteoclasts (Jackson et al., 2006). Consequently, the increased osteoclasts and intensive bone resorption contribute to the pathogenesis of bone loss and osteoporosis.

Previous studies indicated that the differentiation and activation of osteoclasts were regulated by a family of tumor necrosis factor (TNF) like proteins or TNF receptors (TNFRs) including osteoprotegerin (OPG), RANKL and RANK, which co-regulate the function of osteoclasts (Takahashi et al., 1999). Osteoclasts, derived from macrophage/mononuclear precursor cells near or at the surface of bone (Boyle et al., 2003; Normanno et al., 2005), are tissue-specific polynuclear cells and have bone resorption activities (Teitelbaum, 2000). Increased RANKL binding to RANK activates cytoplasmic protein TRAF6, which then activates a series of downstream proteins including IKK, IкB $\alpha$, and NF-אB p65 (Pearson et al., 2001). T cell nuclear factor 1 (NFATc1) and c-Fos, the main transcription factors of osteoclast differentiation, have been reported to be activated by NF- $\mathrm{KB}$ signalling and MAPK pathway such as JNK and ERK (Kim et al., 2012; Lorenzo, 2017). Sequentially, the expression of the osteoclast marker genes related to osteoclast differentiation, such as Atp6v0d2, Acp5, Mmp9, Ctsk and calcitonin receptor (Ctr) are initiated (Boyle et al., 2003). In turn, these pathways synergize to promote osteoclast differentiation and bone resorption. In addition, it was reported that the production of ROS was related to the formation of osteoclasts (Lee et al., 2005).

Natural compounds and their derivatives play an integral role in the development of 
new approaches for the treatment of osteoporosis (Guo et al., 2018). Tiliroside (Tle), extracted from Oriental Paperbush flower, is a kind of flavonoid natural compound with a wide range of pharmacological functions, including anti-inflammatory, anti-thrombotic, antiviral, anti-oxidation and anti-carcinogenic activities (Gao et al., 2017). A recent study demonstrated neuroprotective effects of the compound (Velagapudi et al., 2018). However, there are very few studies on the potential application of Tle in osteoporosis.

In this study, we investigated the role of Tle in osteoclastogenesis and bone loss. We found that Tle inhibited osteoclast formation and bone resorption in vitro, as well as osteoclast marker genes expression and RANKL-mediated MAPK and NFATc1 signalling pathways. Moreover, Tle significantly inhibited the production of reactive oxygen species in cells. Further, Tle attenuated OVX- induced osteoporosis in mice, suggesting that Tiliroside might serve as a new potential therapeutic drug for osteoporosis. 


\section{2 | MATERIALS AND METHODS}

\section{1 | Media and reagents}

Alpha modified minimal essential medium ( $\alpha$-MEM) and FBS were purchased from Thermo Fisher (Scoresby, Australia). Recombinant macrophage colony stimulating factor (M-CSF) was acquired from R\&D Systems (Minneapolis, MN, USA). Tle acquired from Mansite (Chengdu, Sichuan Province, China) was dissolved to $100 \mu \mathrm{M}$ in dimethylsulf-oxide (DMSO) for storage. To make the ratio of DMSO in the total volume less than $0.1 \%, 100 \mu \mathrm{M}$ Tle was then diluted in the culture medium. We purchased antibodies against JNK, p38, p65, NFATc1, phospho-p65, phospho-JNK, phospho-p38 and $\beta$-actin from Cell Signaling Technology (Danvers, MA, USA). Antibody against ERK, phospho-ERK, c-Fos, and IкB $\alpha$ were obtained from Abcam (Cambridge, MA, USA). We acquired MTT Cell Proliferation Assay Kit and Leucocyte acid phosphatase staining kits from Thermo Fisher Scientific (Shanghai, China). We expressed and purified Recombinant GST

rarifatdrduced in the past (Xu et al., 2000). We purchased Reactive Oxygen Species (ROS) Assay Kit from the company, Beyotime Institute of Biotechnology (Shanghai, China).

\section{2 | In vitro osteoclastogenesis assay}

We isolated primary bone marrow-derived macrophages (BMMs) from femur and tibia of 6-week-old mice. In an incubator with $5 \% \mathrm{CO}_{2}$ at $37^{\circ} \mathrm{C}$, BMMs were proliferated in $75 \mathrm{~cm}^{2}$ culture flasks with complete $\alpha$-MEM which was supplemented with 10\% FBS and 1\% penicillin/streptomycin and stimulated with M-CSF (50 ng/ml). When the area of cells reached $90 \%$, they were transferred to a 96-well plate at a density $8 \times 10^{3}$ cells/well and cultured overnight. After attached, the cells were treated with diverse concentrations of Tle $(1.25,2.5,5,10 \mu \mathrm{M})$ in the case of RANKL (50ng/ml) stimulation while we changed the medium every two days until osteoclasts were formed. Then the mature osteoclast-like cells were stained by a leucocyte acid phosphatase staining kit after they were fixed in 4\% paraformaldehyde for 10 minutes and washed with PBS for three times. TRAcP positive multinucleated cells which had more than three nuclei were counted as osteoclasts. The spread area and numbers of 
osteoclasts were counted using ImageJ software.

\subsection{Cell viability assay}

At a density $8 \times 10^{3}$ cells/well, BMMs were planted onto a 96-well plate and cultured overnight. Then various concentrations of Tle $(0,1.25,2.5,5,10,20 \mu \mathrm{M})$ were administrated to the cells. 48 hours later, the cells were given CCK8 solution $(10 \mu \mathrm{l} /$ well) and incubated for another $2 \mathrm{~h}$. By using a microplate reader (Thermo, USA), we detected the absorbance of cells at $450 \mathrm{~nm}$.

\section{4 | F Babtimmunofluorescence and confocal microscopy}

At a density $8 \times 10^{3}$ cells/well, BMMs were planted onto a 96-well plate and cultured overnight. Subsequently, cells were stimulated with $\mathrm{M}$

(50 ng/ml) for 3 days. Next, osteoclast precursor cells were given gradient concentrations of Tle for two days. After being fixed with $4 \%$ paraformaldehyde and permeabilized with $0.1 \%$ Triton $\mathrm{X}$ , t00 ReBS were blocked by 3\% bovine serum albumin in PBS. Next, cells were stained for F belttiafter being incubated in rhodamine froAlugaidedteschahl diadin After being stained with DAPI, the cells were observed by confocal microscopy.

\section{5 | Hydroxyapatite resorption assay}

BMMs were first cultured with complete medium on a 6-well plate, supplemented with M-CSF (50 ng/ml) and RANKL (50 ng/ml). Until mature osteoclasts were formed, they were digested with Tryple reagent (Thermo Fisher Scientific, Shanghai, China) and transferred to 96-well hydroxyapatite coated plates at the same cell density. The cells were continued to be cultured under the stimulation of RANKL while administering different concentrations of Tle $(5,10 \mu \mathrm{M})$ for 48 hours. Cells not treated with Tle were used as a positive control. Half of the wells were counted for the number of multinucleated cells by staining them for TRAcP activity. To wipe out the cells and measure the absorption area, the remaining wells were soaked in $10 \%$ sodium hypochlorite solution for 10 minutes. By using standard light microscope, we 
photographed the resorbed area in each well. By Image J software (NIH, Bethesda, USA), we analyzed the percentage area of the resorbed surface.

\section{6 | Intracellular ROS activity detection}

ROS Assay Kit was applied to the detection of intracellular ROS activity. At a density $8 \times 10^{3}$ cells/well, BMMs were planted onto a 96-well plate. While being stimulated with M-CSF (50 ng/ml) and RANKL (50 ng/ml), the cells were administrated with 5 or $10 \mu \mathrm{M}$ Tle for 3 days. Then cells were incubated with $10 \mu \mathrm{M}$ DCFH-DA in the dark for 30 minutes after being washed with pure $\alpha$-MEM. After that, we used PBS to wash the osteoclast precursor cells and the images were obtained by employing fluorescent microscope.

\section{7| Quantitative real-time PCR}

At a density $1 \times 10^{5}$ cells/well, BMMs were planted onto a 6-well plate. After attached, cells were given Tle $(0,5,10 \mu \mathrm{M})$ while stimulated with M-CSF (50 ng/ml) and RANKL (50 ng/ml) until osteoclast emerged in the positive group. According to the manufacturer's protocol (Thermo Fisher Scientific, Scoresby, Australia), we collected total RNA from cells by using RNA extraction kit. We obtained the cDNA by Moloney murine leukemia virus reverse transcriptase using a q-PCR machine. The q-PCR reaction was performed using the following cycles: $95^{\circ} \mathrm{C}$ for $10 \mathrm{~min}, 40$ cycles which contained $95^{\circ} \mathrm{C}$ for $10 \mathrm{~s}, 60^{\circ} \mathrm{C}$ for 20 sand $72^{\circ} \mathrm{C}$ for $20 \mathrm{~s}$, followed by a final extension at $72^{\circ} \mathrm{C}$ for $90 \mathrm{~s}$. The expression levels of each marker genes were based on the expression of GAPDH and we detected their fold changes using Livak's equation. Primer sequences information for q-PCR was listed in Table 1.

\section{8 | Ethical Use of Animals}

All animal experiments were performed in accordance with the Institutional Animal Ethics Committee of Guangxi Medical University (Guangxi, China). Mice were treated exactly in the light of the principles and procedures of the National Institutes of Health (NIH) Guide for the Care and Use of Laboratory Animals. 


\section{9 | OVX-induced osteoporosis mice model}

Thirty-two (32) 8-week-old C57BL/6 female mice were reared at the Animal Management Center of Guangxi Medical University. Then, they were randomly assigned to four groups (8 mice per group): sham group, OVX group, OVX $+5 \mathrm{mg} / \mathrm{kg}$ Tle group, and OVX $+10 \mathrm{mg} / \mathrm{kg}$ Tle group. Except for the sham group, the bilateral ovaries of the mice in the other groups were surgically removed under anesthesia and the mice were given penicillin once a day for 3 days to prevent any infection. After one week of recovery, the OVX $+5 \mathrm{mg} / \mathrm{kg}$ Tle group and OVX $+10 \mathrm{mg} / \mathrm{kg}$ Tle group were administered intraperitoneally with Tle (5 mg/kg or $10 \mathrm{mg} / \mathrm{kg}$ ) every two days for six weeks, while the other two groups were given the same dose of normal saline as placebo. Subsequently, all mice were sacrificed by excessive anesthesia. To further analyse bone tissue, the tibia was preserved with $4 \%$ paraformaldehyde.

\subsection{0 | Micro-computed tomography (micro-CT) scanning}

The whole tibia was analyzed with anisotropic voxel size of $10.0 \mu \mathrm{m}$ through the Bruker Micro-CT Skyscan 1272 system (Kontich, Belgium). Under the condition of

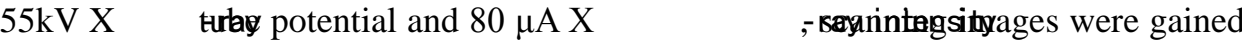
in a 1,700 ms exposure time. The trabecular bone volume per total volume (BV/TV), bone surface density (BS/TV), mean trabecular number (Tb.N) and bone mineral density (BMD) were measured according to previous study (Tornvig et al., 2001).

\subsection{1 | Histomorphometric analysis of bone tissue}

To assess the number of trabecular bone and osteoclasts in the metaphysic of tibias as described formerly (Koide et al., 2009), tibias were decalcified with 10\% EDTA solution for 14 days. The decalcified tibias were then dehydrated by different concentrations of alcohol. Tibias were embedded in paraffin after being cleared twice with xylene. Finally, the embedded tibias were cut into $8 \mu \mathrm{m}$ thick sections for further TRAP staining. 


\subsection{2 | Western blot}

A density $1 \times 10^{6}$ cells/well of BMMs was planted to a 6-well plate. After the cells were attached, they were first starved for 1 hour, then pretreated with Tle $(10 \mu \mathrm{M})$ for 3 hours, and finally stimulated with RANKL (50 ng/ml) for 0, 5, 10, 20, 30 or 60 mins. Total proteins were obtained by using radioimmunoprecipitation (RIPA) lysis buffer to lysis cells. The protein samples were transported onto nitrocellulose (NC) membranes (Thermo Fisher Scientific, Shanghai, China) after being detached through SDS-polyacrylamide gel electrophoresis. Then we used $5 \%$ bovine serum albumin (BSA) to block the membranes for 40 mins. After that, the membranes were gently shaken overnight at $4{ }^{\circ} \mathrm{C}$ with different specific primary antibodies which were diluted to $0.1 \%$ TBST solution. Hereafter, we used $0.1 \%$ TBST to wash the membranes and incubated them in the dark with corresponding species secondary antibodies to which enhanced chemiluminesence reagent (Amersham Pharmacia Biotech, Piscataway, NJ) were added. The proteins were visualised with an Image systent K(ASS HQQ0hcare, Silverwater, Australia).

\subsection{3 | Statistical analysis}

All experimental data which were obtained from three or more independent experiments are presented as mean \pm SD. And the statistical significance was determined using Student's t-test and one-way ANOVA tests. The results with $p<$ 0.05 were considered to be statistically significant. 


\section{3 | RESUTS}

\section{1 | Tle restrained the differentiation of osteoclasts}

The chemical structure of Tle is presented in Figure 1a. In order to test whether Tle has an adverse effect on the proliferation and cytotoxicity of BMMs, BMMs were

given treated with diverse concentrations of Tle $(0,1.25,2.5,5,10,20 \mu \mathrm{M})$ for 48 hours in 96-well plates. According to the result, Tle had no effect on the proliferation of BMMs when the dose of Tle was less than $20 \mu \mathrm{M}$ (Fig. 1b). Therefore, the concentrations of Tle used in this study were not toxic to the cells. To delineate the effect of Tle on osteoclastogenesis, we cultured BMMs in complete medium supplemented with M-CSF and RANKL and then treated them with diverse concentrations of Tle for six days. We observed that the number and size of osteoclasts in the test groups were attenuated and presented a dose-dependent feature compared with the positive control group (Fig. 1c). After TRAcP-positive multinucleated (nuclei $\geq 3$ ) osteoclasts were analyzed for cell number and area ratio, three concentrations at 2.5, 5 and $10 \mu \mathrm{M}$ were statistically significant (Fig. 1d, e).

Next, BMMs were treated with Tle $(10 \mu \mathrm{M})$ at different time periods while being stimulated with RANKL and M-CSF. Figure 2a showed the different time periods of Tle treatment and representative TRAcP staining pictures for osteoclasts. We found that in the early stage (Day 1-3), Tle exerted a more significant inhibition on osteoclast differentiation compared to the positive control group (Fig. 2a). Mature osteoclasts, which were TRAcP-positive and multinucleated (nuclei $\geq 3$ ), were counted in quantity and total area (Fig. 2b, c). Compared with the early stage, Tle exerted a less significant inhibition on osteoclast differentiation in the mid or late stages (Day 3-5 and day 5-6) but the inhibition of osteoclast by Tle was significant in the day 1-6 stage. Taken together, these results showed that Tle restrained osteoclast differentiation induced by RANKL in a dose- and time-dependent manner.

\section{2 | Tle down-regulated the expression levels of marker genes involved in osteoclast differentiation}

To explore the role of Tle in osteoclast differentiation at the genetic level, we 
examined the expression levels of marker genes involved in osteoclast differentiation. BMMs were treated with Tle $(0,5,10 \mu \mathrm{M})$ while being stimulated with RANKL and M-CSF until multinuclear osteoclast-like cells appeared in the positive control group. Then the relative expression levels of marker genes were determined by q-PCR. The results revealed that the expression levels of these marker genes, including Acp5, Mmp9, Atp6v0d2 and Ctsk, were significantly down-regulated in the Tle-treated groups (Fig. 2d, e, f, g).

\section{3 | Tle restrained osteoclastic hydroxyapatite resorption and F-actin belts formation induced by RANKL}

Equal mature osteoclasts were seeded into a 96-well hydroxyapatite coated plates and then the cells were treated with Tle (5 or $10 \mu \mathrm{M}$ ). While compared to the positive group, the hydroxyapatite resorption area decreased in groups treated with Tle (Fig. 3a). According to the TRAcP staining, approximate amount of osteoclasts were counted in each well (Fig. 3b). In addition, we measured the area of bone resorption with Image $\mathrm{J}$ software and found that the area of absorption in the wells treated with Tle was significantly reduced (Fig. 3c). Based on the above results, we could draw a conclusion that Tle reduced the bone-hydroxyapatite resorption area by inhibiting the bone resorption function of osteoclasts rather than reducing the number of osteoclasts. Further, we studied the effect of Tle on the F-actin belts formation which is one of the key actins for osteoclasts-to exert-osteoclastic bone resorption. In the positive control group, polarized F-actin belts were observed (Fig. 1d). However, both the quantity and size of F-actin belts were decreased under the condition of being treated with Tle (Fig. 1d, e).

\section{4 | Tle deincreased intracellular ROS generation in BMMs}

To explore the effect of Tle on intracellular ROS production induced by RANKL, we measured DCF content in each group of BMMs treated with RANKL in the presence or absence of Tle (Fig. 4a). The result revealed that intracellular ROS levels in Tle-treated groups were significantly reduced by Tle when compared to the positive 
group and presented a dose-dependent manner (Fig. 4b). In Figure c, we counted the number of BMMs and found that the number of cells per well was almost equal.

\section{5 | Tle suppressed RANKL-induced activation of MAPK and NFATc1 pathways}

\section{during osteoclastogenesis}

Next, we measured the relative expression of IкB $\alpha$ to $\beta$-actin and phosphorylated p65 to p65 through Western blot assay after osteoclasts were stimulated with RANKL for specific time. Compared to the control group, relative expression of $\operatorname{I\kappa B} \alpha$ and phosphorylated p65 almost did not change in the Tle $(10 \mu \mathrm{M})$ group (Fig. 5a, b, c). In addition, we investigated whether Tle affected MAPK induced by RANKL. Compared to the control, the results indicated that the relative expression of phosphorylated ERK to the total ERK was significantly reduced by the addition of Tle at 10 and 20 minutes (Fig. 5a, d). Furthermore, the relative expression of phosphorylated JNK to the total JNK was obviously decreased at 10, 20 and 30 minutes (Fig. 5a, e). Yet, the relative expression of phosphorylated p38 to total p38 of osteoclasts had little change during each period stimulated with RANKL (Fig. 5a, f).

To examine the effect of Tle on the relative expression levels of NFATc1 and c-Fos which were the pivotal downstream transcription factors, BMMs were cultured with complete medium and stimulated with M-CSF and RANKL for 5 days. Results indicated that the protein expression level of NFATc1 was significantly lower in the Tle-treated groups than that of control group on day 3 and 5. Similarly, the protein expression levels of c-Fos began to decrease on day 1 and 3 compared to the control group (Fig. 5g, h, i). These results indicated an inhibitory effect of Tle on NFATc1 signalling pathway.

\subsection{Tle prevented bone loss in OVX-induced osteoporosis mouse model}

We further studied the potential treatment effects of Tle against osteolytic diseases in vivo by using an osteoporotic OVX model in mice. We scanned the entire tibias of all mice with micro-CT and avoided any damage to the medullary cavity while 
dissociating the tibias from the sacrificed mice to minimise the effects on the results of further histological experiments. It can be seen from the 3-dimensional (3D) reconstructed image of the region of interest of the tibias that the bone mass of the same part of the Tle-treated group was significantly higher compared with the OVX group (Fig. 6a). Compared to the OVX group trabecular BV/TV, trabecular BS/TV, trabecular number (Tb.N) and BMD in Tle-treated groups were significantly higher (Fig. 6b, c, d, e).

Histological analysis further confirmed the results of micro-CT scans. TRAcP staining showed a decrease of osteoclasts in the growth plate of tibia head (Fig. 6f, g, h). In summary, Tle can prevent bone loss in the OVX-induced osteoporosis in mice. 


\section{4 | DISCUSSION}

Osteoporosis, a systemic bone disease characterized by decreased bone mass and destruction of bone tissue microstructure which can bring about increased bone fragility and increased risk of fracture, seriously threatens the health and quality of life of the elderly. Postmenopausal women are the main patients with osteoporosis and a sudden decreased estrogen which contributes to the over-activation of osteoclast is the major motivator of postmenopausal osteoporosis (Rachner et al., 2011). However, osteoporosis is preventable and treatable. So far, drugs for the treatment of osteoporosis mainly include two major categories: inhibition of bone resorption drugs including calcium, vitamin D and active vitamin D, calcitonin, diphosphate, estrogen, etc. and promotion of bone formation drugs including synthetic steroids, parathyroid hormone, etc. (Khosla and Hofbauer, 2017). In recent years, studies on the prevention of osteoporosis by small molecule Chinese herbal medicines have increased significantly because they have the advantage of weak side effects (Chen et al., 2017; Guo et al., 2018; Ma et al., 2018). Tle, a flavonoid natural compound extracted from Oriental Paperbush flower, has been reported to postpone the process of cellular senescence (Chatzigeorgiou et al., 2017), inhibit the oxidation of low density lipoprotein (Schinella et al., 2007), and contribute to neuroprotective function and antihypertensive effects etc. (Silva et al., 2013; Velagapudi et al., 2018). However, the effect of Tle on osteoclasts and the specific mechanisms are still unclear.

Coordination of the functions of osteoclasts and osteoblasts is essential to maintain the balance of bone homeostasis in natural physiology (de Baat et al., 2005). Once this balance is broken, various bone-related diseases will occur. For example, the bone resorption function of over-activated osteoclast can lead to severe bone structural damage in Paget disease, RA, and osteoporosis (Cornish et al., 2002; Ma et al., 2013; Meier et al., 2006). Decreased estrogen leads to excessive activation of osteoclasts. In this study, we demonstrated that Tle prevented bone loss in OVX-induced osteoporosis mouse model. Then we used a series of experiments to identify the effects of Tle on osteoclast differentiation and bone resorption in vitro. The number of 
osteoclasts was significantly reduced by Tle during RANKL-induced osteoclast differentiation. When we planted the same number of osteoclasts on hydroxyapatite plate, it was shown that Tle reduced the hydroxyapatite bone-resorption area by inhibiting the bone-resorption function of osteoclasts rather than reducing the number of osteoclasts. In addition, Tle significantly inhibited the formation of F-actin belts which contributed to osteoclast bone resorption. Tle down-regulated the expression of Ctsk and Atp6v0d2 which were involved in the regulation of F-actin belts formation and bone resorption function in osteoclasts (Dodds et al., 2001; Tanaka et al., 2013; Wilson et al., 2009). Moreover, our q-PCR assay also found that the expression of genes participated in osteoclast differentiation had been down-regulated, including Acp5, Mmp9, Atp6v0d2, and Ctsk.

NF- $\mathrm{BB}$ signalling pathway, one of the classical pathways for osteoclast differentiation, is a targeted pathway for various drugs to treat osteolytic bone disease (Brown et al., 2008). RANKL and M-CSF are two pivotal factors in inducing BMMs to differentiate into mature osteoclasts and a human monoclonal antibody against RANKL was used to treat osteoporosis (Kearns et al., 2008). After RANKL binds to RANK, TRAF6 is activated and phosphorylates IKK and I $\mathrm{KB} \alpha$ in turn, causing p65 to enter the nucleus, ultimately leading to the expression of osteoclast-related genes which are drawn into osteoclast differentiation and its cellular function. Activation-Degradation of IкB $\alpha$ and entry of p65 into the nucleus are two key points events of the NF- $\kappa B$ pathway activation in osteoclast formation (Xu et al., 2009). In this study, the expression levels of p-65 and IkB $\alpha$ did not change in Tle-treated osteoclasts compared to the untreated control. Therefore, believed-it is likely that Tle did not affect differentiation of osteoclasts through the NF-kB pathway. Hence, another pathway-MAPK was considered. The MAPK pathway contains three major activation pathways: JNK, p38 and ERK (Choi et al., 2017). Activated Ras leads to sequential activation of Raf and MEK1/2, further increasing phosphorylated-ERK. Increased phosphorylated- ERK up-regulated the downstream expression level of c-Fos and NFATc1. Similarly, JNK and p38 are separately activated by MKK4/7 and MKK3/6, which results in increased 
phosphorylated jnk and p38. The increase of phosphorylated JNK and ERK leads to the increasing expression level of downstream such as NFATc1 and c-Fos, leading to osteoclast differentiation. We found that the relative expression of phosphorylated ERK to the total ERK and phosphorylated JNK to the total JNK were obviously inhibited by Tle. Therefore, the MAPK pathway was considered to be the pivotal pathway for Tle to inhibit osteoclast differentiation.

NFATc1 and c-Fos proteins, the two crucial downstream proteins of MAPK pathways, can activate the expression of markers related to osteoclast differentiation including TRAcP (Acp5), Mmp9, Atp6v0d2 and cathepsin K (Ctsk) etc. (Feng et al., 2009; Matsuo et al., 2004). From the result of Western blot, the relative expression levels of NFATc1 and c-Fos were inhibited by Tle. This just explained the decreased expression of marker genes associated with osteoclast differentiation. Furthermore, ROS production was reported to have a pivotal effect on osteoclast differentiation and MAPK signalling activation (Lee et al., 2005; Yip et al., 2005). So we further confirmed that the production of ROS was reduced by Tle. Those results indicated that Tle suppressed RANKL-induced activation of MAPK (mainly JNK and ERK) pathway in osteoclastogenesis and ROS production. However, whether Tle directly affect the MAPK pathway or inhibit the production of ROS, which indirectly affects the MAPK signalling pathway remains to be addressed. And we will further explore theThe specific molecular mechanism of Tle inhibiting ROS production in thewill require future researchfurther investigation.

In summary, Tle was confirmed to have significant inhibitory effects on osteoclast differentiation in vitro and prevent bone loss in the OVX model mainly through MAPK and NFATc1 signaling pathways. Moreover, research on Tle-derived drugs is of great significance for the treatment of esteoporosis -bone diseases such as osteoporosis. 


\section{ACKNOWLEDGMENTS}

It is supported by the National Natural Science Foundation of China (81501910), the Natural Science Foundation of Guangxi Province (2015GXNSFCA414001, 2015GXNSFDA139019), the Guangxi Collaborative Innovation Center for Biomedicine talent cultivation (GCICB-TC-2017001) and the Australian Health and Medical Research Council (NHMRC, APP1107828, APP1027932, APP1163933).

\section{CONFLICTS OF INTEREST}

The authors declare that they have no conflicts of interest. 


\section{REFERENCES}

Bono CM, Einhorn TA. 2003. Overview of osteoporosis: pathophysiology and determinants of bone strength. European spine journal : official publication of the European Spine Society, the European Spinal Deformity Society, and the European Section of the Cervical Spine Research Society 12 Suppl 2:S90-96.

Boyle WJ, Simonet WS, Lacey DL. 2003. Osteoclast differentiation and activation. Nature 423(6937):337-342.

Brown KD, Claudio E, Siebenlist U. 2008. The roles of the classical and alternative nuclear factor-kappaB pathways: potential implications for autoimmunity and rheumatoid arthritis. Arthritis Res Ther 10(4):212.

Chatzigeorgiou S, Thai QD, Tchoumtchoua J, Tallas K, Tsakiri EN, Papassideri I, Halabalaki M, Skaltsounis AL, Trougakos IP. 2017. Isolation of natural products with anti-ageing activity from the fruits of Platanus orientalis. Phytomedicine 33:53-61.

Chen X, Zhi X, Pan P, Cui J, Cao L, Weng W, Zhou Q, Wang L, Zhai X, Zhao Q, Hu H, Huang B, Su J. 2017. Matrine prevents bone loss in ovariectomized mice by inhibiting RANKL-induced osteoclastogenesis. FASEB journal : official publication of the Federation of American Societies for Experimental Biology 31(11):4855-4865.

Choi JH, Han Y, Kim YA, Jin SW, Lee GH, Jeong HM, Lee HS, Chung YC, Lee YC, Kim EJ, Lee KY, Jeong HG. 2017. Platycodin D Inhibits Osteoclastogenesis by Repressing the NFATc1 and MAPK Signaling Pathway. J Cell Biochem 118(4):860-868.

Cornish J, Callon KE, Bava U, Lin C, Naot D, Hill BL, Grey AB, Broom N, Myers DE, Nicholson GC, Reid IR. 2002. Leptin directly regulates bone cell function in vitro and reduces bone fragility in vivo. The Journal of endocrinology 175(2):405-415.

de Baat P, Heijboer MP, de Baat C. 2005. [Development, physiology, and cell activity of bone]. Nederlands tijdschrift voor tandheelkunde 112(7):258-263.

Dodds RA, James IE, Rieman D, Ahern R, Hwang SM, Connor JR, Thompson SD, Veber DF, Drake FH, Holmes S, Lark MW, Gowen M. 2001. Human osteoclast cathepsin K is processed intracellularly prior to attachment and bone resorption. J Bone Miner Res 16(3):478-486.

Feng H, Cheng T, Steer JH, Joyce DA, Pavlos NJ, Leong C, Kular J, Liu J, Feng X, Zheng MH, Xu J. 2009. Myocyte enhancer factor 2 and microphthalmia-associated transcription factor cooperate with NFATc1 to transactivate the V-ATPase d2 promoter during RANKL-induced osteoclastogenesis. J Biol Chem 284(21):14667-14676.

Gao D, Fu QF, Wang LJ, Wang DD, Zhang KL, Yang FQ, Xia ZN. 2017. Molecularly imprinted polymers for the selective extraction of tiliroside from the flowers of Edgeworthia gardneri (wall.) Meisn. J Sep Sci 40(12):2629-2637.

Guo Q, Cao Z, Wu B, Chen F, Tickner J, Wang Z, Qiu H, Wang C, Chen K, Tan R, Gao Q, Xu J. 2018. Modulating calcium-mediated NFATc1 and mitogen-activated protein kinase deactivation underlies the inhibitory effects of kavain on osteoclastogenesis and bone resorption. J Cell Physiol 234(1):789-801.

Jackson RD, Wactawski-Wende J, LaCroix AZ, Pettinger M, Yood RA, Watts NB, Robbins JA, Lewis CE, Beresford SA, Ko MG, Naughton MJ, Satterfield S, Bassford T. 2006. Effects of conjugated equine estrogen on risk of fractures and BMD in postmenopausal women with hysterectomy: results from the women's health initiative randomized trial. J Bone Miner Res 21(6):817-828.

Kearns AE, Khosla S, Kostenuik PJ. 2008. Receptor activator of nuclear factor kappaB ligand and 
osteoprotegerin regulation of bone remodeling in health and disease. Endocr Rev 29(2):155-192.

Khosla S, Hofbauer LC. 2017. Osteoporosis treatment: recent developments and ongoing challenges. Lancet Diabetes Endocrinol 5(11):898-907.

Kim HJ, Yoon KA, Lee MK, Kim SH, Lee IK, Kim SY. 2012. A novel small molecule, NecroX-7, inhibits osteoclast differentiation by suppressing NF-kappaB activity and c-Fos expression. Life Sci 91(19-20):928-934.

Koide M, Kinugawa S, Ninomiya T, Mizoguchi T, Yamashita T, Maeda K, Yasuda H, Kobayashi Y, Nakamura H, Takahashi N, Udagawa N. 2009. Diphenylhydantoin inhibits osteoclast differentiation and function through suppression of NFATc1 signaling. J Bone Miner Res 24(8):1469-1480.

Lee NK, Choi YG, Baik JY, Han SY, Jeong DW, Bae YS, Kim N, Lee SY. 2005. A crucial role for reactive oxygen species in RANKL-induced osteoclast differentiation. Blood 106(3):852-859.

Lorenzo J. 2017. The many ways of osteoclast activation. The Journal of clinical investigation 127(7):2530-2532.

Ma C, Xu K, Meng J, Ran J, Adel Abdo Moqbel S, Liu A, Yan S, Wu L. 2018. Tectorigenin inhibits RANKL-induced osteoclastogenesis via suppression of NF-kappaB signalling and decreases bone loss in ovariectomized C57BL/6. J Cell Mol Med 22(10):5121-5131.

Ma XN, Ge BF, Chen KM, Zhou J, Shi WG, Xie YF, Guo XY, Lv X, Cheng K, Gao YH. 2013. [Mechanisms of icariin in regulating bone formation of osteoblasts and bone resorption of osteoclasts]. Zhongguo yi xue ke xue yuan xue bao Acta Academiae Medicinae Sinicae 35(4):432-438.

Matsuo K, Galson DL, Zhao C, Peng L, Laplace C, Wang KZ, Bachler MA, Amano H, Aburatani H, Ishikawa H, Wagner EF. 2004. Nuclear factor of activated T-cells (NFAT) rescues osteoclastogenesis in precursors lacking c-Fos. J Biol Chem 279(25):26475-26480.

Meier C, Meinhardt U, Greenfield JR, De Winter J, Nguyen TV, Dunstan CR, Seibel MJ. 2006. Serum cathepsin $\mathrm{K}$ concentrations reflect osteoclastic activity in women with postmenopausal osteoporosis and patients with Paget's disease. Clinical laboratory 52(1-2):1-10.

Normanno N, De Luca A, Aldinucci D, Maiello MR, Mancino M, D'Antonio A, De Filippi R, Pinto A. 2005. Gefitinib inhibits the ability of human bone marrow stromal cells to induce osteoclast differentiation: implications for the pathogenesis and treatment of bone metastasis. Endocr Relat Cancer 12(2):471-482.

Pearson G, English JM, White MA, Cobb MH. 2001. ERK5 and ERK2 cooperate to regulate NF-kappaB and cell transformation. J Biol Chem 276(11):7927-7931.

Rachner TD, Khosla S, Hofbauer LC. 2011. Osteoporosis: now and the future. Lancet 377(9773):1276-1287.

Reventlow S, Bang H. 2006. Brittle bones: ageing or threat of disease exploring women's cultural models of osteoporosis. Scand J Public Health 34(3):320-326.

Schinella GR, Tournier HA, Manez S, de Buschiazzo PM, Del Carmen Recio M, Rios JL. 2007. Tiliroside and gnaphaliin inhibit human low density lipoprotein oxidation. Fitoterapia 78(1):1-6.

Silva GC, Pereira AC, Rezende BA, da Silva JP, Cruz JS, de Souza Mde F, Gomes RA, Teles YC, Cortes SF, Lemos VS. 2013. Mechanism of the antihypertensive and vasorelaxant effects of the flavonoid tiliroside in resistance arteries. Planta Med 79(12):1003-1008.

Takahashi N, Udagawa N, Suda T. 1999. A new member of tumor necrosis factor ligand family, ODF/OPGL/TRANCE/RANKL, regulates osteoclast differentiation and function. Biochemical 
and biophysical research communications 256(3):449-455.

Tanaka H, Tanabe N, Kawato T, Nakai K, Kariya T, Matsumoto S, Zhao N, Motohashi M, Maeno M. 2013. Nicotine affects bone resorption and suppresses the expression of cathepsin K, MMP-9 and vacuolar-type $\mathrm{H}(+)$-ATPase d2 and actin organization in osteoclasts. PLoS One 8(3):e59402.

Teitelbaum SL. 2000. Bone resorption by osteoclasts. Science 289(5484):1504-1508.

Tornvig L, Mosekilde LI, Justesen J, Falk E, Kassem M. 2001. Troglitazone treatment increases bone marrow adipose tissue volume but does not affect trabecular bone volume in mice. Calcif Tissue Int 69(1):46-50.

Velagapudi R, El-Bakoush A, Olajide OA. 2018. Activation of Nrf2 Pathway Contributes to Neuroprotection by the Dietary Flavonoid Tiliroside. Mol Neurobiol 55(10):8103-8123.

Wilson SR, Peters C, Saftig P, Bromme D. 2009. Cathepsin K activity-dependent regulation of osteoclast actin ring formation and bone resorption. J Biol Chem 284(4):2584-2592.

Xu J, Tan JW, Huang L, Gao XH, Laird R, Liu D, Wysocki S, Zheng MH. 2000. Cloning, sequencing, and functional characterization of the rat homologue of receptor activator of NF-kappaB ligand. J Bone Miner Res 15(11):2178-2186.

Xu J, Wu HF, Ang ES, Yip K, Woloszyn M, Zheng MH, Tan RX. 2009. NF-kappaB modulators in osteolytic bone diseases. Cytokine Growth Factor Rev 20(1):7-17.

Yip KH, Zheng MH, Steer JH, Giardina TM, Han R, Lo SZ, Bakker AJ, Cassady Al, Joyce DA, Xu J. 2005. Thapsigargin modulates osteoclastogenesis through the regulation of RANKL-induced signaling pathways and reactive oxygen species production. I Bone Miner Res 20(8):1462-1471.

Yoshimura N, Muraki S, Oka H, Kawaguchi H, Nakamura K, Akune T. 2010. Cohort profile: research on Osteoarthritis/Osteoporosis Against Disability study. Int J Epidemiol 39(4):988-995. 
Figure legends

Fig. 1 Tle restrained the differentiation of osteoclasts in a dose-dependent manner. (a) The chemical structure of Tle. (b) Cell viability was measured by MTT assay. (c) BMMs were stimulated with complete medium containing RANKL (50 $\mathrm{ng} / \mathrm{ml})$ and M-CSF (50 ng/ml) in the presence of different concentrations of Tle (0, 1.25, 2.5, 5, $10 \mathrm{uM}$ ). Until osteoclasts were formed, they were stained for TRAcP staining. (d) TRAcP-positive cells with more than three nuclei were counted as osteoclasts. (e) Spread area of TRAcP-positive cells with more than three nuclei. All data were confirmed in 3 independent experiments. Data are expressed as means \pm SD. ${ }^{*} \mathrm{p}<0.05,{ }^{* *} \mathrm{p}<0.01,{ }^{* * *} \mathrm{p}<0.001$ relative to the control group. Scale bar presents $200 \mathrm{uM}$

Fig. 2 Tle restrained RANKL-induced osteoclast differentiation in a time-dependent manner and down-regulated the expression levels of marker genes involved in osteoclast differentiation. (a) BMMs were stimulated with complete medium containing RANKL (50 ng/ml) and M-CSF (50 ng/ml) and separately treated with Tle (10 uM) in day 1-3, 3-5 and 5-6 while the positive group was not treated with Tle. Then the cells were stained for TRAcP staining. (b, c) The number and spread area of mature osteoclasts were counted. (d, e, f, g) Osteoclast marker genes expression levels were determined. All data were confirmed in 3 independent experiments. Data are expressed as means $\pm \mathrm{SD} .{ }^{*} \mathrm{p}<0.05,{ }^{* *} \mathrm{p}<0.01$, ${ }^{* * *} \mathrm{p}<0.001$ relative to the control group. Scale bar presents $200 \mathrm{uM}$.

Fig. 3 Tle restrained osteoclastic hydroxyapatite resorption and F-actin belt formation induced by RANKL. (a) Representative images of TRAcP stained osteoclasts on hydroxyapatite coated surfaces and hydroxyapatite resorption. (b, c) The number of osteoclasts and the area of the bone resorption pit were counted by ImageJ software. (d) BMMs were stimulated with $\mathrm{M}$ (50 $\mathrm{ng} / \mathrm{ml}$ ) for 3 days and treated with gradient concentrations of Tle for two days. 
confocal microscopy. (e) F-actin belt area was quantified._All data were confirmed in 3 independent experiments. Data are expressed as means \pm SD. ${ }^{*} \mathrm{p}<0.05,{ }^{* * *} \mathrm{p}<$ 0.001 relative to the control group. Scale bar presents $200 \mathrm{uM}$.

Fig. 4 Tle increased intracellular ROS generation in BMMs. (a) Representative microscopic fields of osteoclast precursor cells were shown. (b) Average DCF intensity per cell presented the average ROS production per cell. (c) BMMs per well were counted. All data were confirmed in 3 independent experiments. Data are expressed as means $\pm \mathrm{SD}$. ${ }^{* *} \mathrm{p}<0.01$ relative to the control group. Scale bar presents $200 \mathrm{uM}$.

Fig. 5 Tle suppressed RANKL-induced activation of MAPK and NFATc1 pathways during osteoclastogenesis. (a) BMMs were stimulated with RANKL (50 $\mathrm{ng} / \mathrm{ml}$ ) for $0,5,10,20,30$ or 60 mins after being pretreated with Tle or DMSO for 3 hours. Total cell lysates was analyzed by Western Blotting assay using specific antibodies. (b, c, d, e, f) The relative phosphorylation levels of ERK, JNK, p65, p38 and the relative expression of IкB $\alpha$ were determined and quantified by ImageJ software. (g, h, i) Similarly, BMMs were treated with Tle or DMSO while stimulated with RANKL (50 ng/ml) for $0,1,3,5$ days. Total cell lysates was analyzed by Western Blotting. The relative expression levels of NFATc1 and c-Fos were determined and quantified by ImageJ software. All data were confirmed in 3 independent experiments. Data are expressed as means $\pm \mathrm{SD} .{ }^{*} \mathrm{p}<0.05,{ }^{* *} \mathrm{p}<0.01$ relative to the control group.

Fig. 6 Tle prevented bone loss in OVX-induced osteoporosis mouse model. (a) Representative 3-dimensional micro-CT reconstruction images of the 4 groups at 7 weeks postsurgery. (b, c, d, e) Micro-CT analyses of BV/TV, BS/TV, and Tb.N in the interest region and BMD. (f) Representative images of TRAcP staining for proximal tibia in each group were given. (g, h) The ratio of spread area of osteoclasts to bone area and the number of osteoclasts of the interest region in each group were analyzed 
by ImageJ software $(\mathrm{n}=3)$. Data are expressed as means \pm SD. ${ }^{*} \mathrm{p}<0.05,{ }^{* *} \mathrm{p}<0.01$, ${ }^{* * *} \mathrm{p}<0.001$ relative to the control group.

Fig. 7 Tle suppressed NFATc1 and c-Fos expression through attenuating phosphorylation of JNK and ERK, while leaving p-38 and NF-KB unaffected. 
a

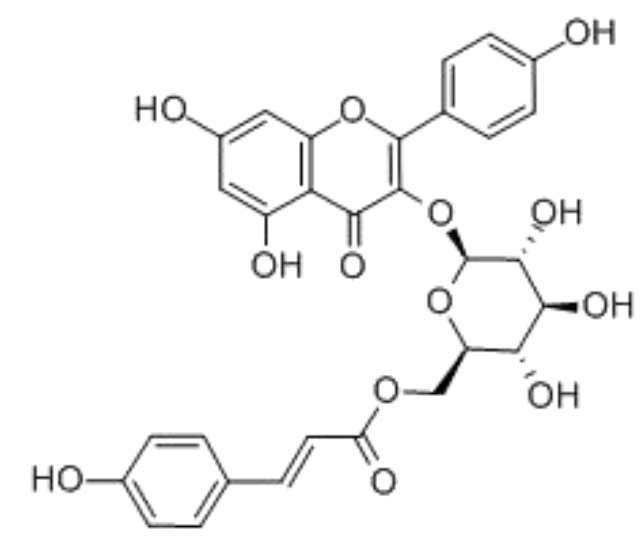

Tle: $\mathrm{C} 30 \mathrm{H} 26 \mathrm{O} 13$ b

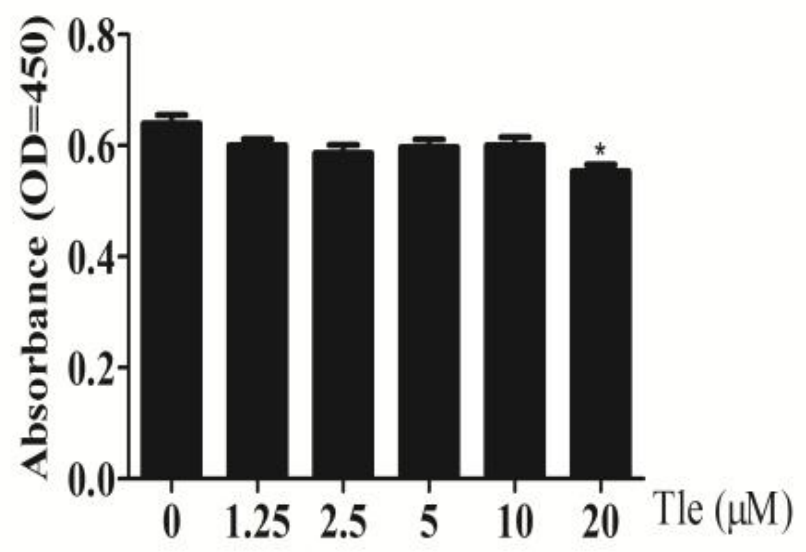

C

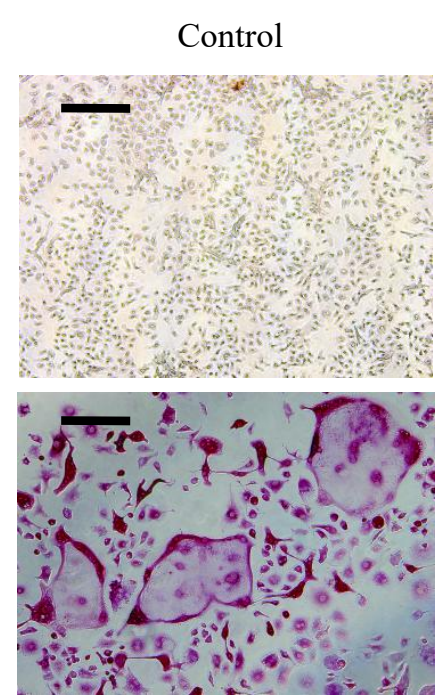

RANKL $+2.5 \mu \mathrm{M}$ Tle
RANKL

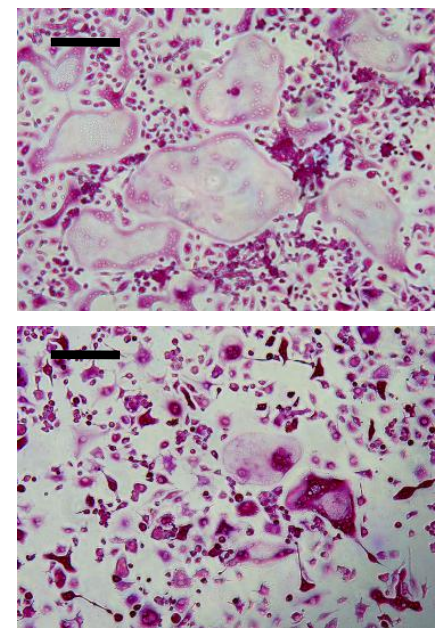

RANKL $+5 \mu \mathrm{M}$ Tle
RANKL $+1.25 \mu \mathrm{M}$ Tle
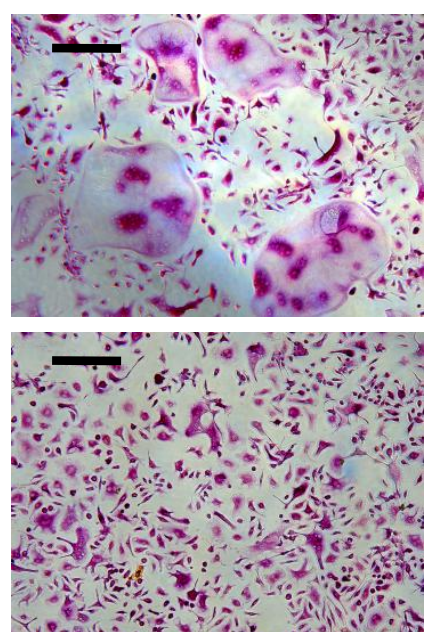

RANKL $+10 \mu \mathrm{M}$ Tle d

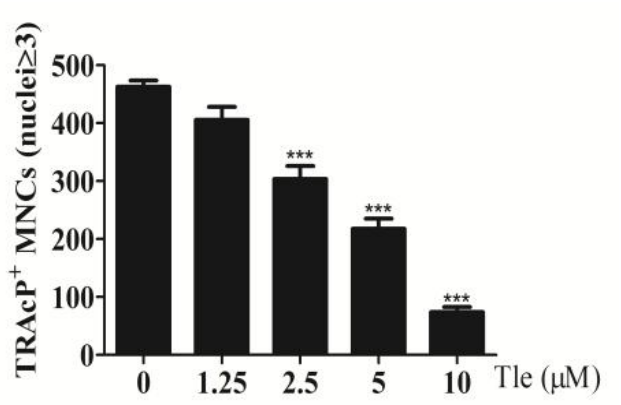

e

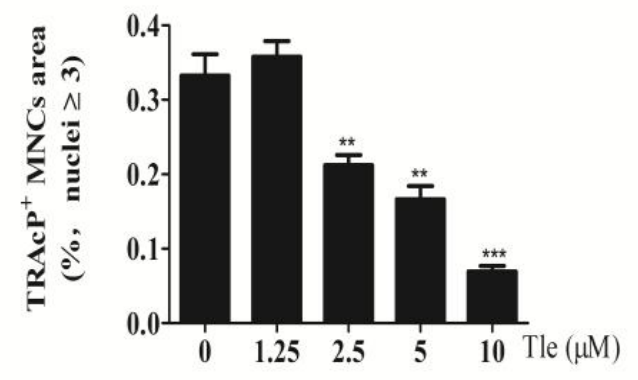


a

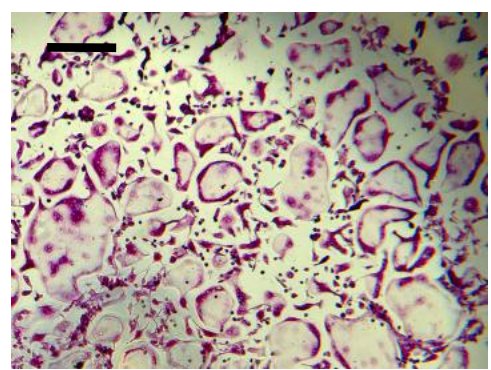

Tle (-)

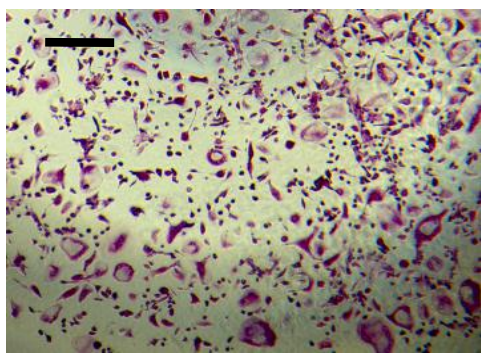

10 $\mu \mathrm{M}$ Tle Day 1-3

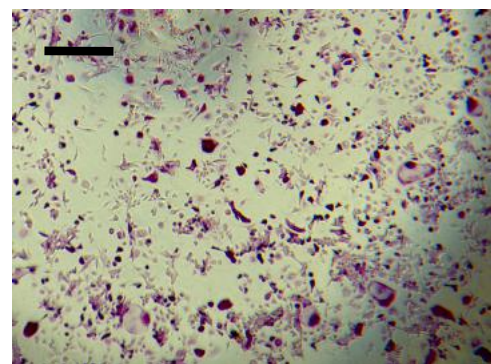

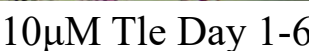

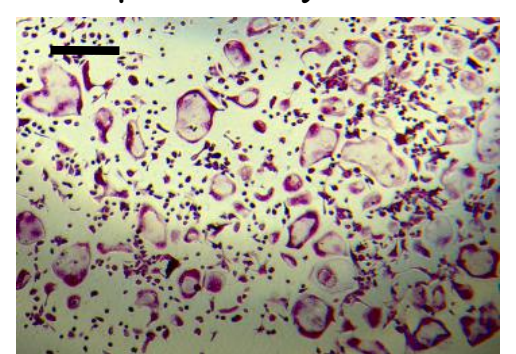

10 $\mu \mathrm{M}$ Tle Day 3-5

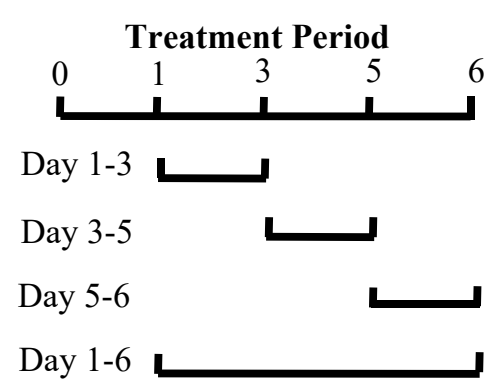

Day 1-6

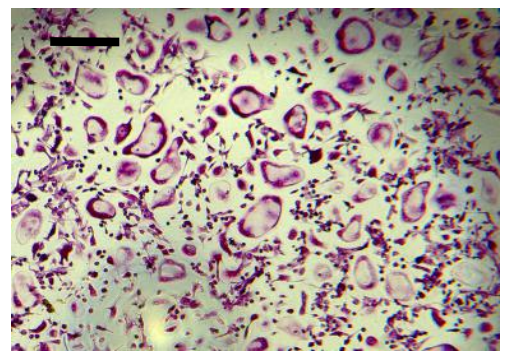

$10 \mu \mathrm{M}$ Tle Day 5-6 b

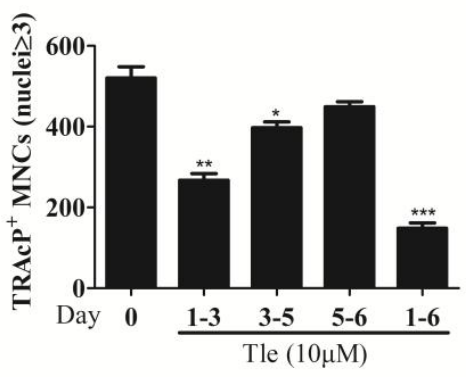

c

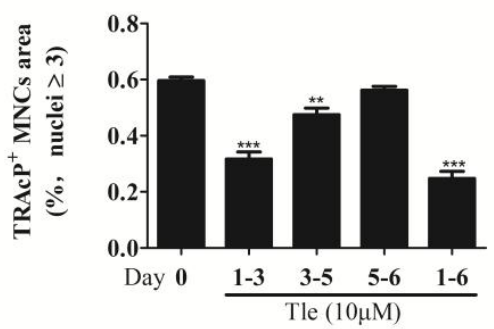

g

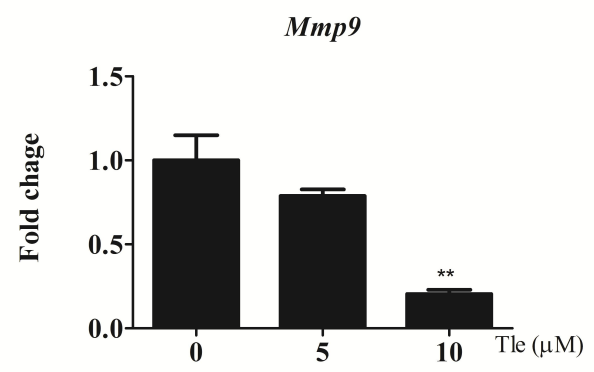

d

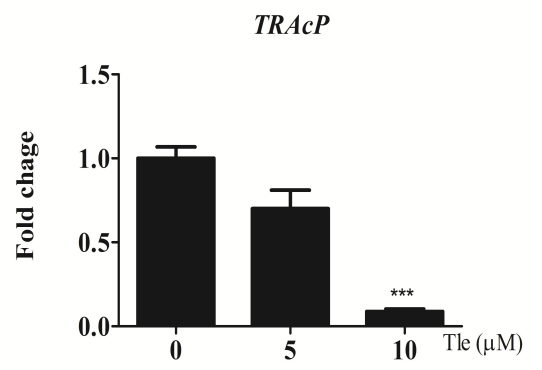

e

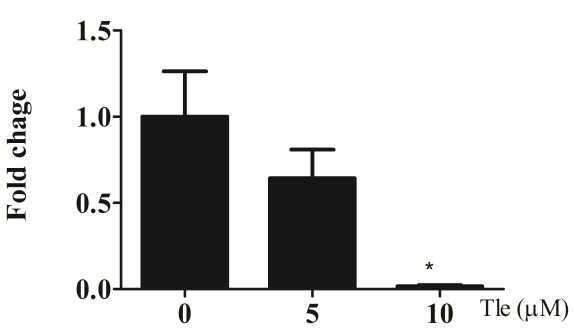

f

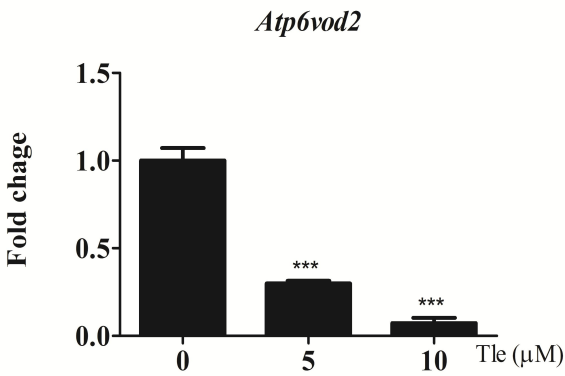


$\mathrm{a}$
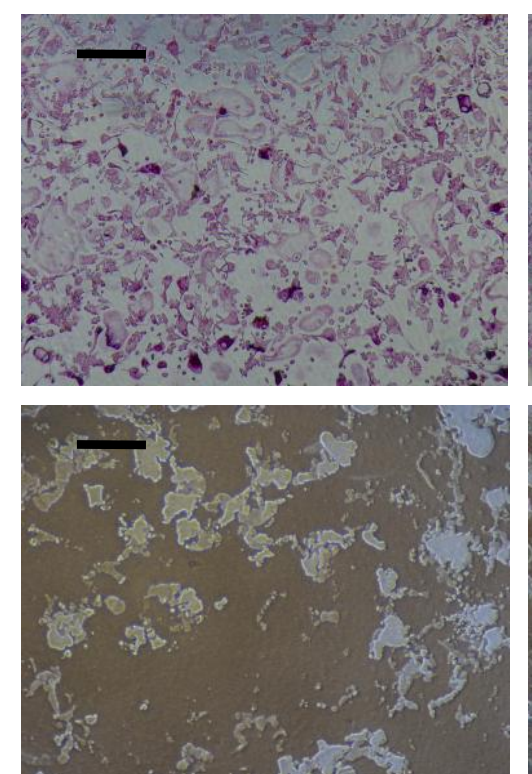

d

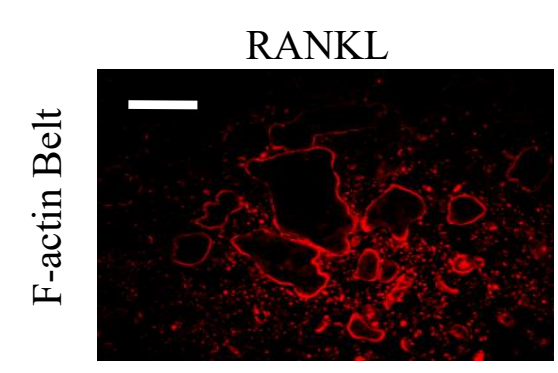

$\frac{1}{2}$

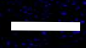

$\frac{\overline{0}}{3}$

$\sum^{\circ}$
RANKL $+5 \mu \mathrm{M}$ Tle
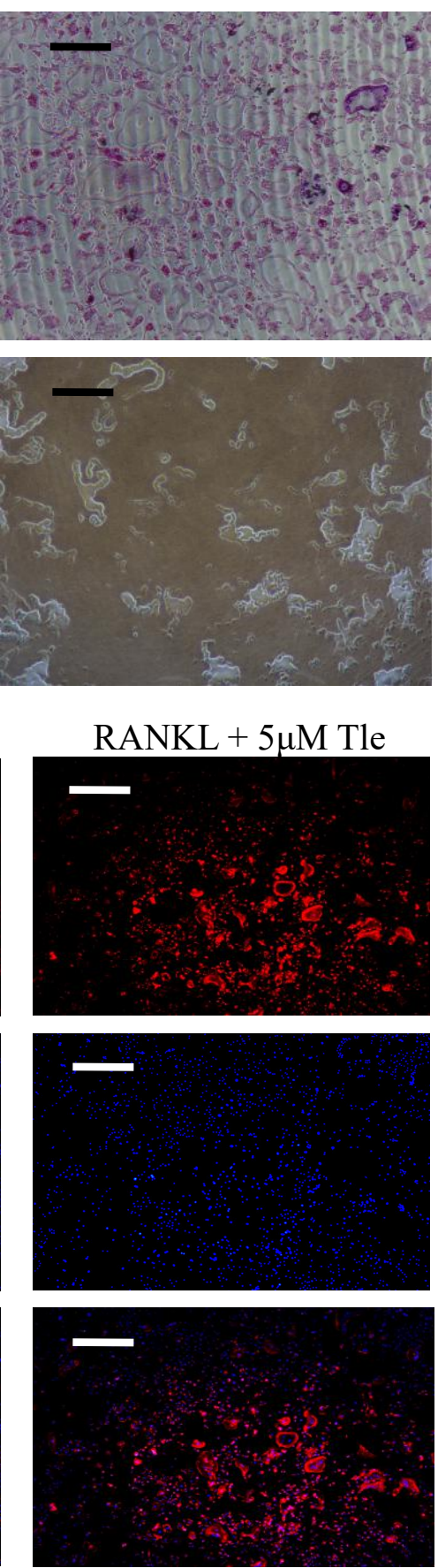

RANKL $+10 \mu \mathrm{M}$ Tle
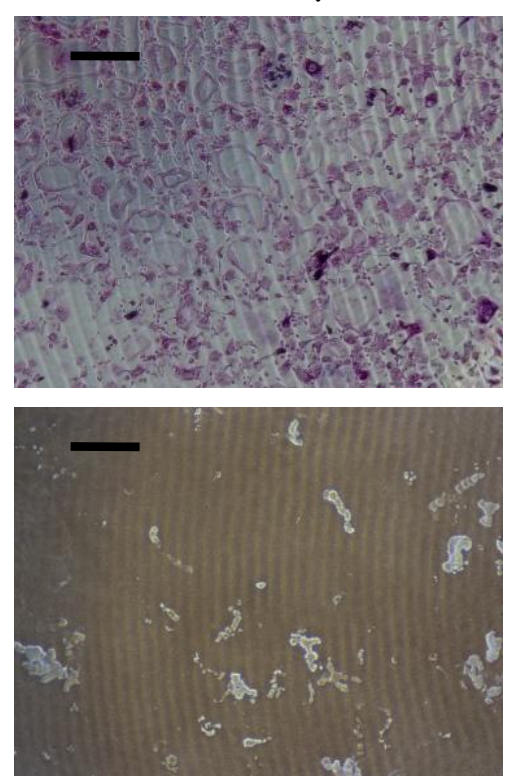

RANKL $+10 \mu \mathrm{M}$ Tle
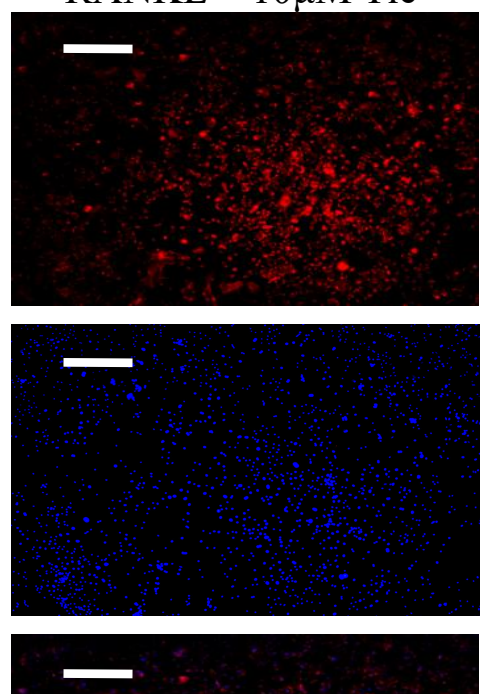

b

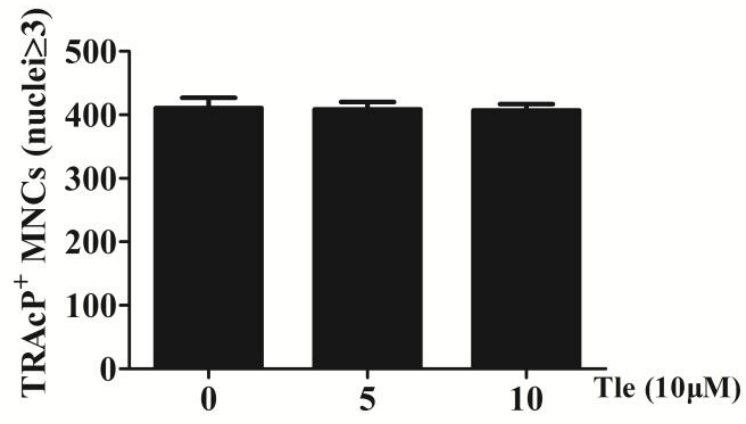

c

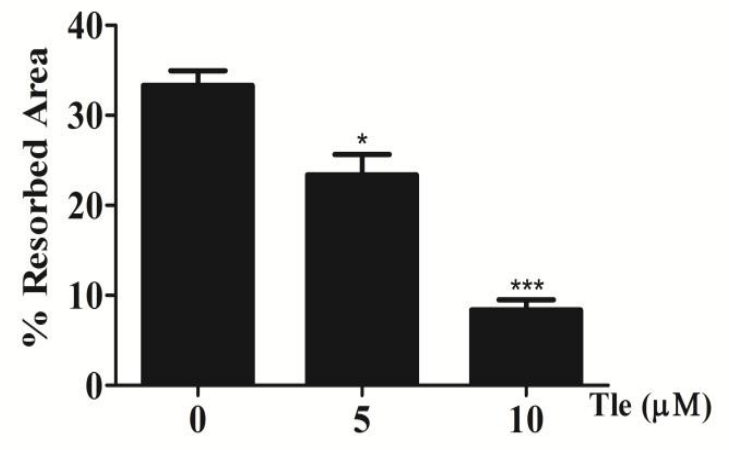

e

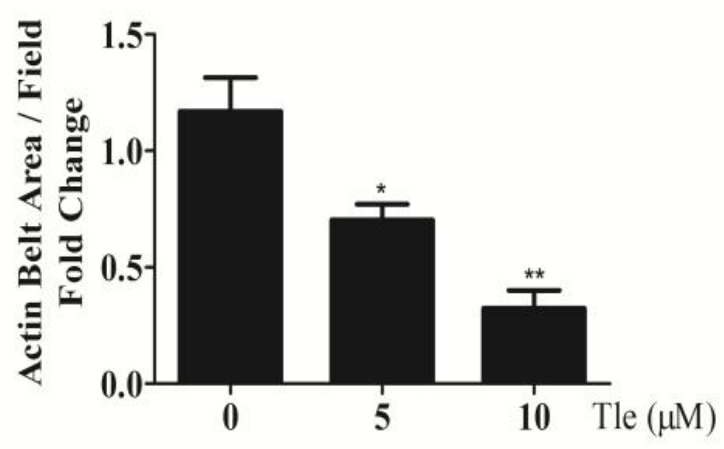




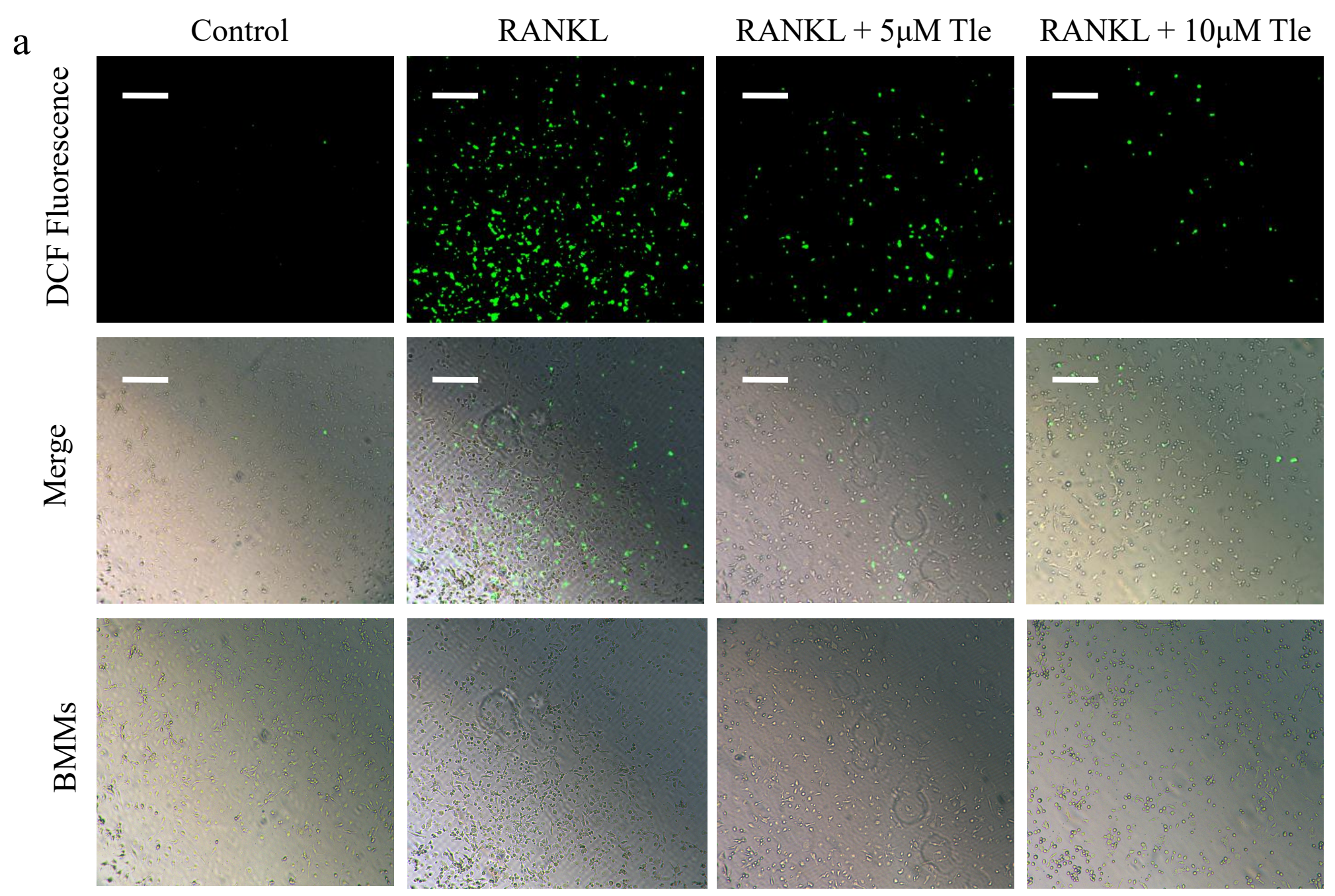

b

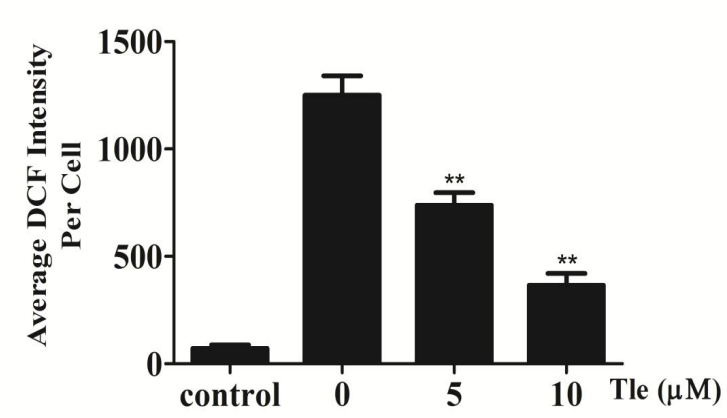

c

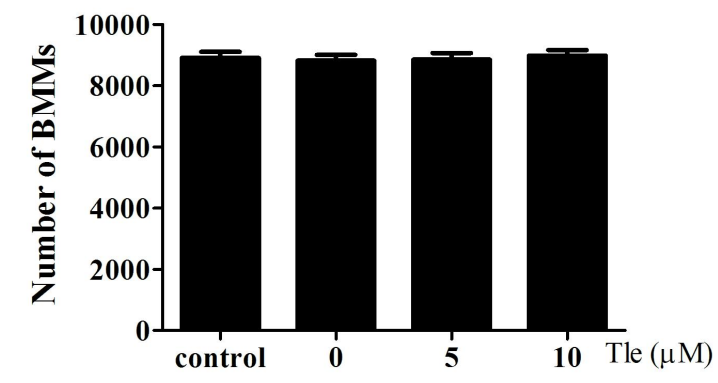


a

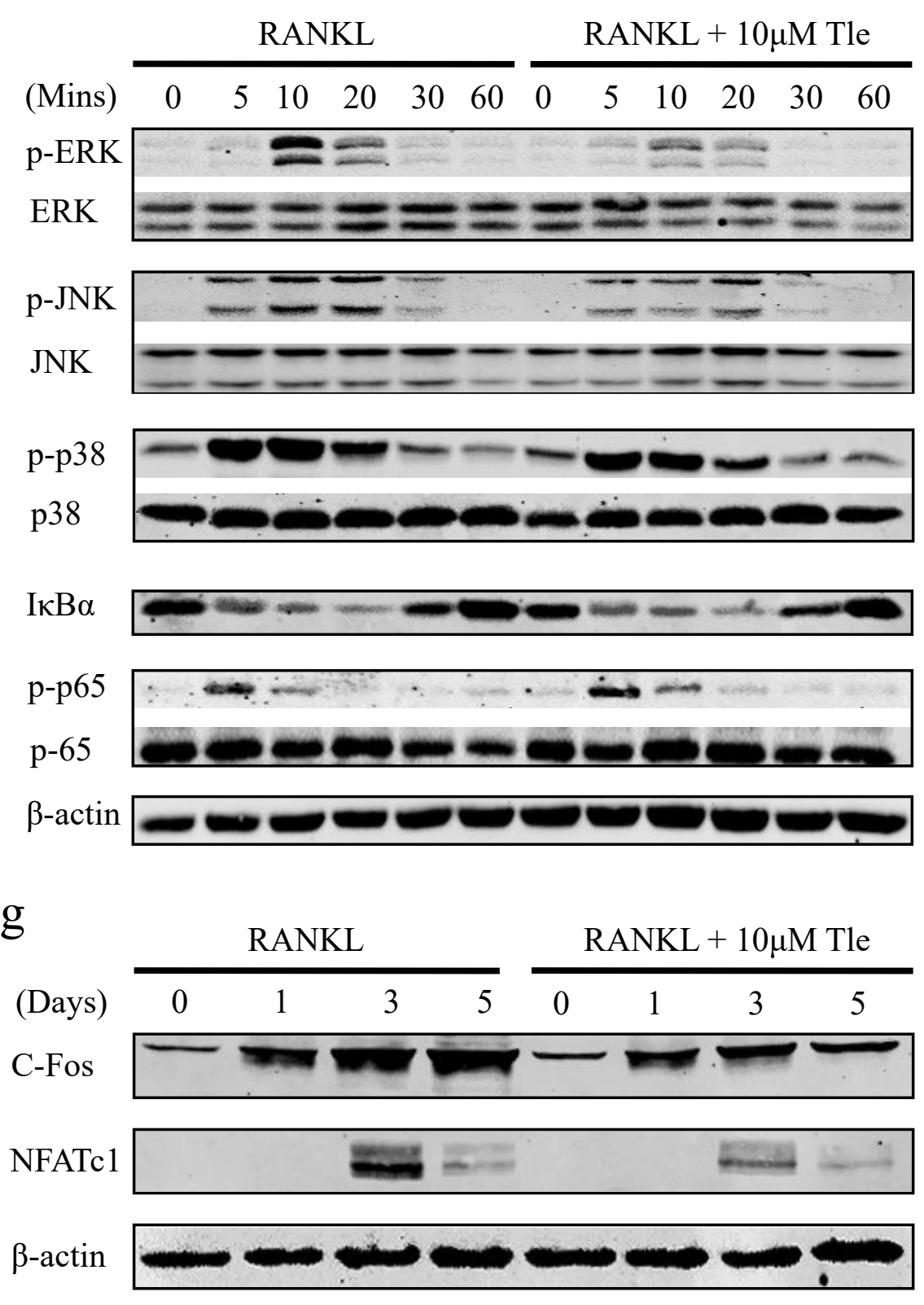

b

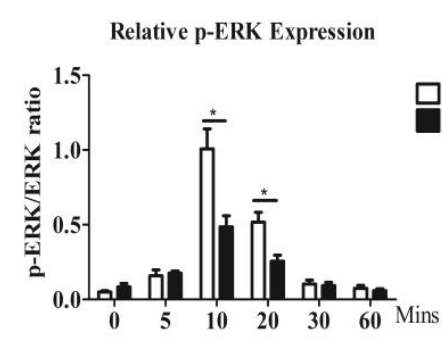

d

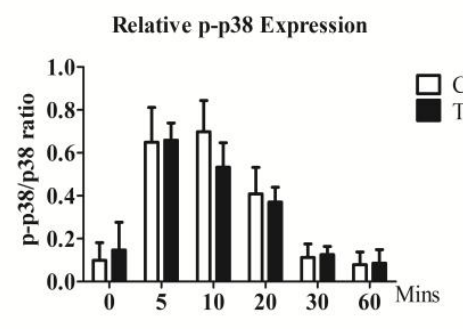

$\mathrm{f}$

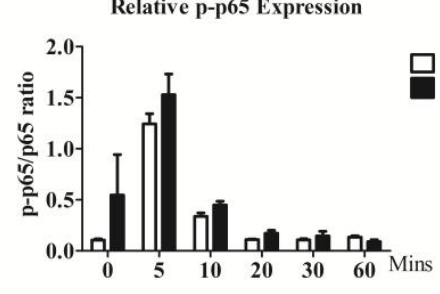

h

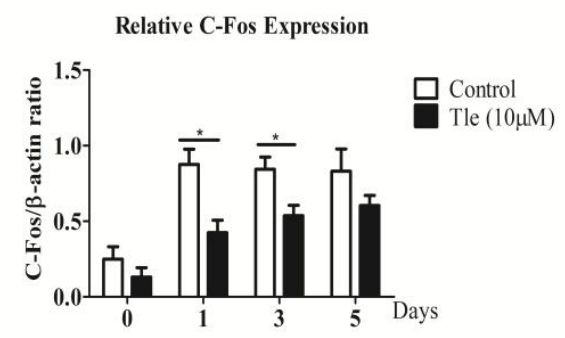

C

Relative p-JNK Expression

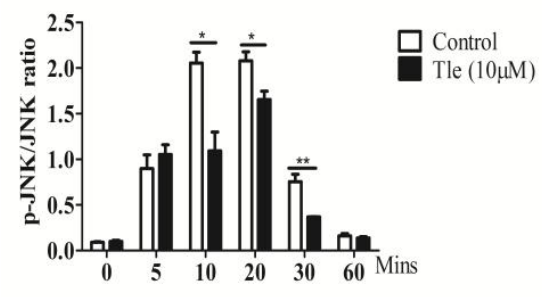

e

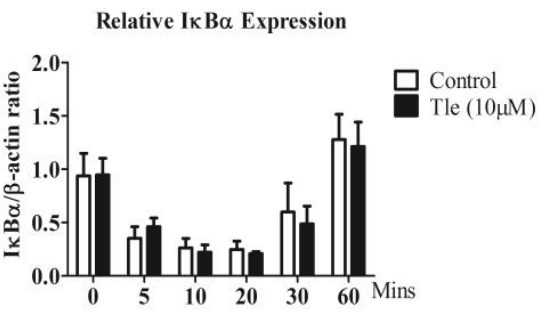

i

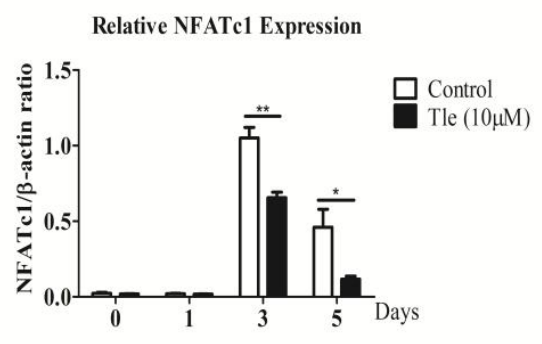




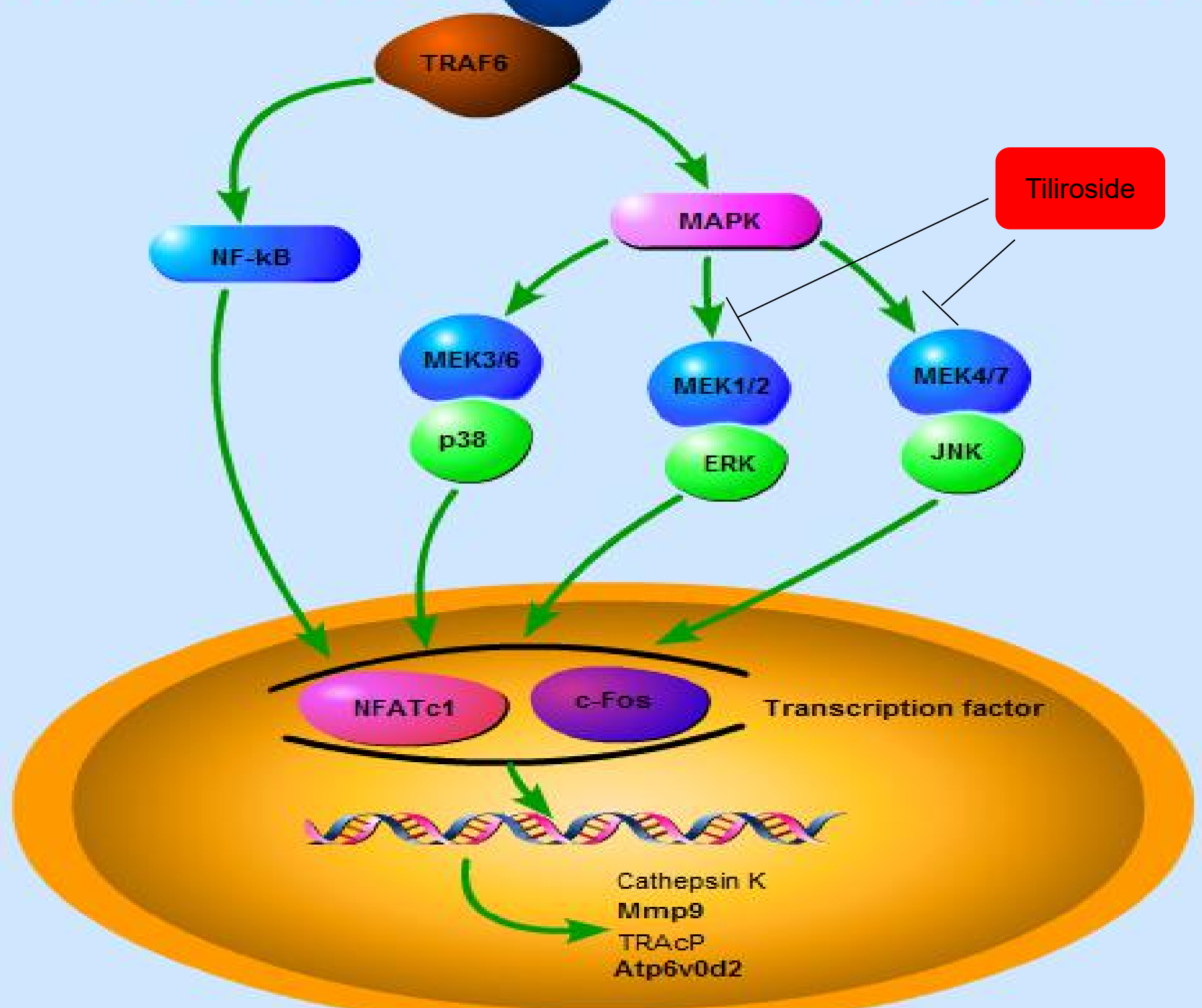

